\title{
Overview of the ATLAS data acquisition system operating at the $\mathrm{TeV}$ scale
}

\author{
Claudia Borer on behalf of the ATLAS Collaboration
}

\begin{abstract}
This paper focuses on the operation of the ATLAS data acquisition system during the first months of 2010. ATLAS is one of the two multipurpose detectors at the Large Hadron Collider (LHC), which provides proton-proton collisions at the unprecedented centre-of-mass energy of $7 \mathrm{TeV}$. The ATLAS data acquisition system is based on $O(2 k)$ processing nodes, interconnected by a multi-layer Gigabit Ethernet network. About $20 \mathrm{k}$ applications will provide the needed capabilities in terms of run control, event selection, data flow, local storage and data monitoring.

The whole data acquisition system has been successfully commissioned during the last two years with cosmic ray and calibration data and it turned out to be robust and reliable. Nevertheless, the continuous operation with beams, the concurrent trigger commissioning, and the understanding of detector and physics performance will pose new challenges. The flexibility of the data acquisition infrastructure will be probed and exploited, in order to comply with the consequent unpredictable working conditions in terms of data-flow, monitoring and configuration requirements. Concerning the latter in particular, the data acquisition efficiency will have to be kept under control, profiting by the special tools and techniques especially put in place. The goal is to minimise both downtime and dead-time, allowing for runtime reconfiguration of the data acquisition and sub-detectors systems as well as for automatic error handling and recovery.
\end{abstract}

\section{INTRODUCTION}

A TLAS is one of the two general purpose experiments to exploit the physics discovery potential of the LHC (Large Hadron Collider) at CERN (European Organization for Nuclear Research). Cross-sections of interesting physics processes at the LHC are highly suppressed with respect to the total cross-section, as shown in Fig. 1. With proton-proton collisions at the centre-of-mass energy of $14 \mathrm{TeV}$, precision measurements like the leptonic $W$ decay are suppressed by $O\left(10^{-7}\right)$, and new physics, as for example the Higgs boson production, are suppressed by $O\left(10^{-10}\right)$.

This high suppression means that, at nominal luminosity of $10^{34} \mathrm{~cm}^{-2} \mathrm{~s}^{-1}$ with a bunch crossing rate of $40 \mathrm{MHz}$ and the typical ATLAS event size of $\sim 1.6 \mathrm{MB}$, the ATLAS detector would produce $64 \mathrm{~TB} / \mathrm{s}$ of mostly non-interesting data. The trigger and data acquisition (TDAQ) system is designed to handle the extremely high data rates.

\section{ATLAS TRIgGER AND DATA ACQUisition (TDAQ)}

The trigger and data acquisition system of ATLAS consists of a three-level trigger architecture. The level 1 (LVL1) is a hardware trigger, whereas the level 2 (LVL2) and the last filter,

Claudia Borer is with the Albert Einstein Center for Fundamental Physics, Laboratory for High Energy Physics, University of Bern, Bern, Switzerland (e-mail: claudia.borer@cern.ch).

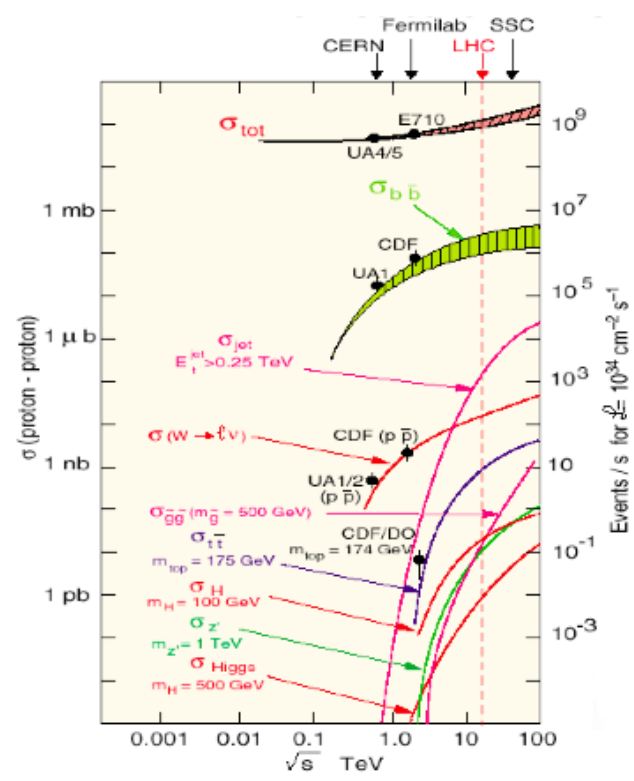

Fig. 1. Production cross-sections of various processes of interest at the LHC as a function of the p-p centre-of-mass energy.

called the event filter (EF), are based on software algorithms. LVL2 and EF form the High Level Trigger (HLT) [2], [3]. A schematic view of the ATLAS TDAQ is shown in Fig. 2.

The LVL1 performs the event selection at $75 \mathrm{kHz}$, corresponding to a bandwidth of $110 \mathrm{~GB} / \mathrm{s}$. The maximum latency allowed is $2.5 \mu \mathrm{s}$. LVL1 uses coarse calorimeter and muon detector information. Based on this information, the LVL1 defines Regions of Interest (RoI) and sends them on to LVL2. The DAQ system uses RoIs to reduce the data-flow requirements. An RoI is a slice of the detector's readout, in the $\eta$ - $\phi$ plane $^{1}$, which contains the fraction of the event that resulted in the LVL1 trigger (Fig. 3). Further trigger decisions are based on this information as guidance and in addition, tracking information will be available for the higher trigger levels.

The data gets buffered in pipelines on the detector. Depending on the LVL1 result, an event either drops off at the end of the pipeline, or gets pushed out via optical fibres to the Read-Out Drivers (ROD). In the RODs the raw data from the detector front end electronics get assembled in event fragments and are moved on for temporary buffering in the Read-Out Buffers (ROB). The ROBs are connected via optical links to the RODs. The Read-Out System (ROS) hosts about 550 custom made boards (so called ROBIN cards, see [4]), each equipped with

\footnotetext{
${ }^{1}$ azimuthal angle $\tan \phi=\frac{p_{y}}{p_{x}}$, pseudo-rapidity $\eta=-\ln \tan \frac{\theta}{2}$ with $\tan \theta=\frac{p_{z}}{p_{T}}$
} 


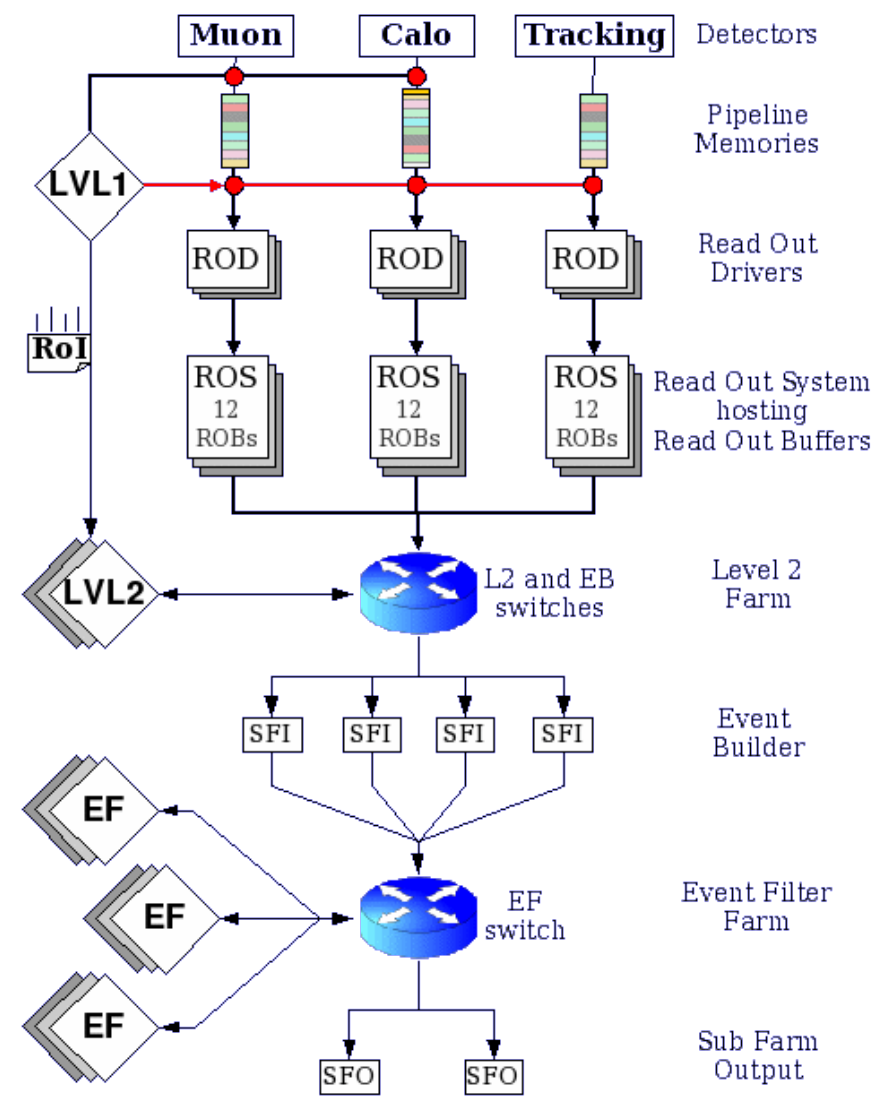

Fig. 2. Schematic view of the TDAQ system.

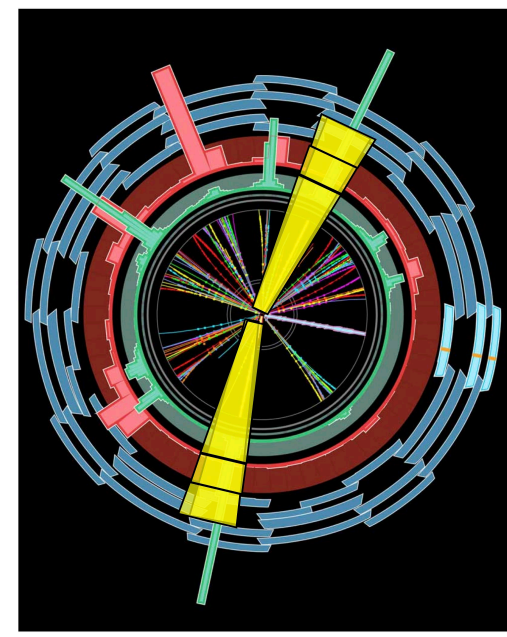

Fig. 3. $\eta-\phi$ plane of the detector. Yellow slices: Regions of Interest.

3 ROBs, which makes a total of about 1600 ROBs. The ROS is a push-pull system. Data are pushed from the detector into the ROBs, where they stay buffered. Other subsystems later in the data-flow will pull the data out by sending a request to the ROS.

The LVL2 receives the RoIs and the LVL1 result. It performs a local analysis within the RoI and thus refines the LVL1 result, with a mean processing time of $40 \mathrm{~ms}$. To do so, LVL2 requests data fragments from the ROS system. In average, it uses only about $2 \%$ of the full event information. The LVL2

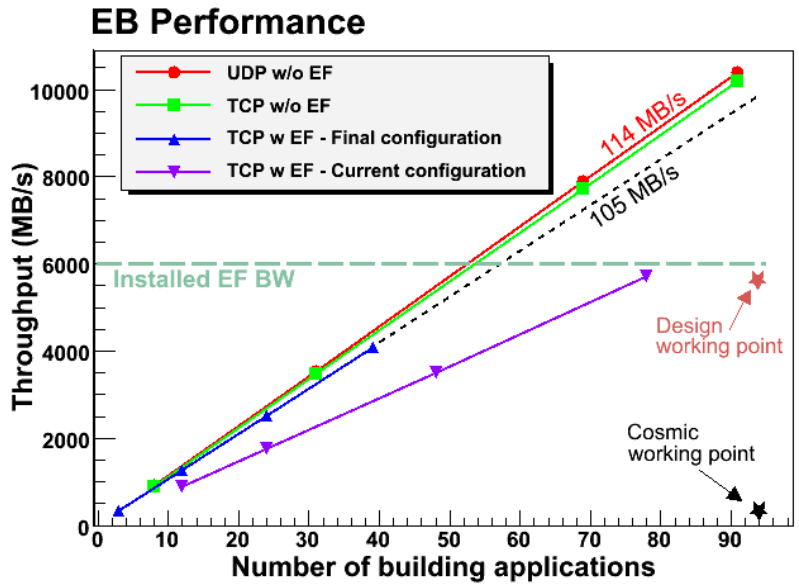

Fig. 4. Performance of the event building system. The red and the green line show the EB performance without the EF, exploiting the whole 90 SFIs, using two different network protocols. The blue line shows the throughput obtained with the EF, taking into account the final PCs configuration. Since not all the foreseen $\mathrm{EF}$ nodes have been deployed yet, the experimental points stop and the line has been extrapolated. The violet line shows the performance which can be obtained nowadays exploiting the SFIs.

trigger reduces the rate from $75 \mathrm{kHz}$ down to about $3 \mathrm{kHz}$. As such, the bandwidth is brought down from $\sim 110 \mathrm{~GB} / \mathrm{s}$ to $4.5 \mathrm{~GB} / \mathrm{s}$. The usage of RoIs is an effective rejection mechanism which imposes less stringent requirements on the ROS system and the networking, in terms of bandwidth and CPU power.

The LVL2 decision is transferred to the event builder system (EB). In about 100 Sub-Farm Input (SFI) applications running on the event builder nodes, the events get fully assembled. This happens at the LVL2's accept rate of $\sim 3 \mathrm{kHz}$. A special feature of the EB is the so called partial event building. Even during standard physics runs, events for sub-detector calibration purposes can be processed. These calibration events do not require the information from the whole ATLAS detector, but just from the specific sub-detector ones, according to the specific need of the calibration purpose. The reduced event size for calibration events allows for a higher LVL2 accept rate, while keeping the total bandwidth constant.

Fig. 4 shows that the EB has been successfully validated during test runs in 2009. The throughput of the EB scales linearly with the number of SFIs. Currently we are running 94 SFI applications on 63 EB nodes. The performance is limited by the installed EF network bandwidth of $6 \mathrm{~GB} / \mathrm{s}$. This is about half of the bandwidth intended for the final EF system. With an event size of 1.6 MB, this is beyond the design EB rate.

The EF accesses the fully assembled events from the SFIs, meaning it operates the trigger menu on the full event data. The mean processing time is around $4 \mathrm{~s}$. The rate is further reduced to $\sim 200 \mathrm{~Hz}$, corresponding to a bandwidth of 300 $\mathrm{MB} / \mathrm{s}$. Accepted events are then sent on for local TDAQ storage to the Sub-Farm Output (SFO) nodes.

The HLT farm currently consists of 850 nodes, configured in such a way that the nodes can either be used as a LVL2 or EF node (the so called XPU nodes, standing for interchangeable 
processing unit). The typical distribution in early 2010 data taking is 250 nodes for LVL2, and 600 nodes for the EF.

In the SFOs, data are written into files and then sent on for permanent storage for offline reconstruction to CASTOR (CERN central mass storage facility [5]). At this stage, the data is sorted into streams, e.g. physics, calibration, express. A stream itself consists of several type specific streams, for instance, the physics stream can be subdivided into jets, muons and B-physics, electrons and photons, etc. If an event satisfies more than one streaming condition, it gets copied and thus adds to the overall output bandwidth. The main motivation for streaming is extra flexibility for prioritised offline reconstruction [6].

The SFO farm consists of 6 nodes, 5 for production plus 1 hot spare. An SFO holds 3 RAID-5 disc controllers, each supervising 8 discs. This gives a total area of 10.5 TB per SFO. The three independent RAID arrays maximise the throughput. The total storage capacity of $52.5 \mathrm{~TB}$ corresponds to a disc buffer for two days of data taking in case of mass storage failure. The nominal value of the SFO system is $300 \mathrm{MB} / \mathrm{s}$ at $200 \mathrm{~Hz}$. The sustained I/O rate of the SFO farm is $550 \mathrm{MB} / \mathrm{s}$, with a peak rate of more than $700 \mathrm{MB} / \mathrm{s}$.

\section{THE CURRENT TDAQ SYSTEM}

The current numbers of nodes for each data-flow subsystem are shown in Table I. Note the numbers for LVL2 and the EF are the shared XPUs of the HLT farm. Further nodes are going to be installed in June 2010. At the moment the HLT consists of $35 \%$ of the expected final system, with $\mathrm{O}(10 \mathrm{k})$ software processes running on all the nodes. For LVL2 there are eight applications running per node, giving a total of 2000. The same applies for the EF nodes, summing up to 4800 applications. The EB is currently running 100 SFI applications. The ROS, SFI, and SFO farms are completely installed.

Comparing the software requirements at design and now, there are also major differences to be noted. The design foresaw $\mathrm{O}(1 \mathrm{k})$ software processes for the whole control tree. Currently there are $\mathrm{O}(10 \mathrm{k})$ software processes running for controlling the system. This is due to the new multicore technology which was not known at the time of design, resulting in one process running on every core. A Finite State Machine (FSM) stears the system. It was supposed to provide a fast start and stop for a change of run, and to configure to load new settings. With the highly increased number of processes, the state transitions are slow and not perfectly reliable, and to change conditions takes more time than initially foreseen. The start taking an average time of $110 \mathrm{~s}$, the stop transition lasts in average $170 \mathrm{~s}$, and the configure state even lasts $260 \mathrm{~s}$ in average.

By design, events should be rejected as early as possible. The current working point of the data flow rejects events as late as possible (in the EF). This is due to the ongoing commissioning of the triggers. There is also a minor change in the event size. The 1.6 MB of the design are slightly reduced to $1.5 \mathrm{MB}$ due to the partial event building claiming some of the bandwidth. With the HLT selection algorithms being commissioned, the data taking is aimed at recording as many collision events as the SFOs can handle. By design the SFOs write data to
TABLE I

OVERVIEW OF THE NUMBER OF NODES OF THE CURRENT DATA-FLOW SYSTEM. THE 850 NODES OF THE HLT FARM (LVL2 AND EF) ARE INTERCHANGEABLE UNITS (XPU) WHICH CAN EITHER BE USED AS LVL2 OR EF.

\begin{tabular}{|l|c|c|}
\hline & Current & Final \\
\hline \hline ROS & 153 & 153 \\
\hline LVL2 & 250 (XPUs) & 500 \\
\hline EB & 63 & 63 \\
\hline EF & 600 (XPUs) & 1800 \\
\hline SFO & 6 & 6 \\
\hline
\end{tabular}

TABLE II

OUTPUT RATES OF THE THREE TRIGGER LEVELS.

\begin{tabular}{|l|c|c|}
\hline & Design & Current \\
\hline \hline LVL1 & $75 \mathrm{kHz}$ & $4 \mathrm{kHz}$ \\
\hline LVL2 & $2-3 \mathrm{kHz}$ & $2.5 \mathrm{kHz}$ \\
\hline EF & $200 \mathrm{~Hz}$ & $200 \mathrm{~Hz}$ \\
\hline
\end{tabular}

tape at a maximum of $300 \mathrm{MB} / \mathrm{s}$. The SFOs though are used beyond design specifications, and reach a sustained $\mathrm{I} / \mathrm{O}$ rate of $550 \mathrm{MB} / \mathrm{s}$. The expected and measured rates for the various trigger levels are shown in Table II.

\section{DATA TAKING}

A primary interest of the ATLAS collaboration is to minimise the down-time and optimise the data taking efficiency during the not yet regular LHC working cycle. Therefore, automatic procedures for a fast and reliable error recovery as well as for a fast start-up have been put in place:

- the stopless removal

- the stopless recovery

- the dynamic resynchronisation

- the warm start/stop

If parts of the read-out are causing the trigger to stop completely, the stopless removal mechanism allows to remove these faulty parts without having to stop the run. After a stopless removal, a resynchronisation is necessary. The dynamic resynchronisation procedure puts these parts back in synchronisation with the LHC clock without having to stop. After the resynchronisation follows the stopless recovery. The expert system framework is automatically notified and takes care of re-enabling the faulty channels.

The dynamic resynchronisation can also be used as a preventive method. An ATLAS channel can lose the synchronisation with the LHC clock. The dynamic resynchronisation allows to recover from this situation before a trigger stop has been provoked. This is faster than having to run through the whole stopless removal/recovery procedures.

The idea of the "warm start" is to start the ATLAS data taking as soon as stable beams for physics studies are circulating within the LHC. Not all the ATLAS components can be powered at the nominal voltage before the LHC beams get into stable conditions. For example, the silicon pixel detector [1], located at few centimeters distance from the beam pipe, could be seriously damaged by astray proton radiations. Once 


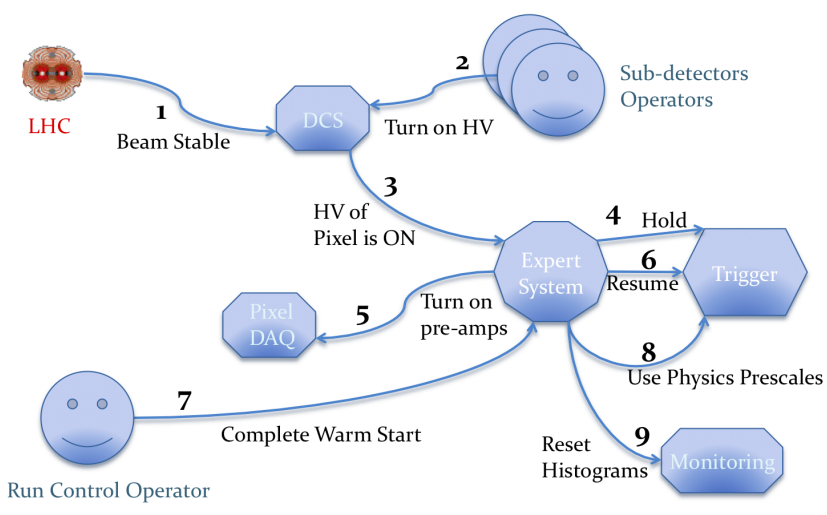

Fig. 5. Warm start. If stable beam is imminent, the detector is switched on. The expert system automatically takes care of trigger and histogram changes.

stable beam condition has been established, the expert system automatically enforces the final detectors to start up. All the detectors are eventually brought to nominal working conditions by the operator. The expert system then automatically sets the trigger configuration for selecting interesting events. Different trigger configurations can be applied depending on the desired physics studies. A similar procedure is used for the "warm stop" in case the beams become unstable. The schematic process sequence is shown in Fig. 5 .

The live-time, in which there is stable beam and ATLAS is taking data, is calculated as follows:

$$
\text { eff }=\frac{T_{\text {RunBeam }}-T_{\text {deadtime }}}{T_{\text {Beam }}},
$$

where $T_{\text {Beam }}$ is the total period with colliding stable beams, $T_{\text {RunBeam }}$ is the actual running time while having beam, and $T_{\text {deadtime }}$ is the fraction of time in which we are not taking data. The latter can happen due to several reasons, such as a change of trigger prescales or busy channels in the system.

In Fig. 6 the data taking efficiency is shown for a period of 43 days. The width of the grey bars is a measure of stable beam availability during 24 hours. The green bars correspond to an average efficiency calculated for a period of 24 hours. So the absence of filled bars indicates a period of no stable beams. Calculating the live-time for the data taking period in April 2010 , we get an overall efficiency of $96.5 \%$.

\section{CONCLUSION}

The ATLAS three-level trigger architecture takes advantage of the use of regions of interest to reduce the data-flow requirements. It is a highly distributed system with $\sim 3000$ nodes. One third of the high-level trigger farm is installed, more machines are coming within the next few months. As the commissioning of the triggers is currently ongoing, events are rejected as late as possible, as opposed to the design. The level 1 trigger output rate is low, currently at $\mathrm{O}(1 \mathrm{kHz})$, due to the start-up mode operations. There are automatic procedures in place to maximise the live-time. Overall data taking efficiency is excellent.

\section{REFERENCES}

[1] ATLAS Collaboration, The ATLAS Experiment at the CERN Large Hadron Collider, JINST 3 (2008) S08003.

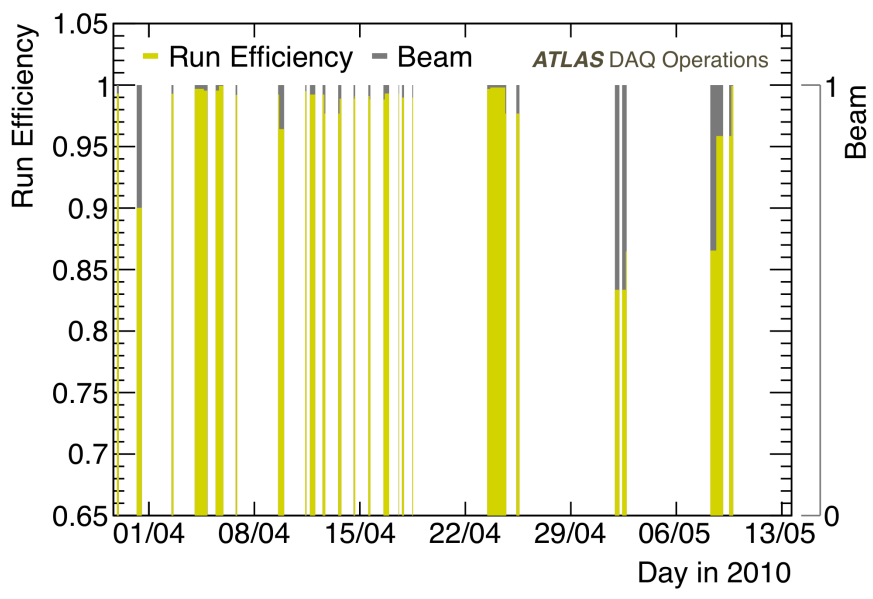

Fig. 6. The data taking efficiency, defined as the ratio of running time during beam time to beam time, is shown. The beam time is defined by the presence of two circulating stable beams. The running time incorporates the fraction of dead time. The width of the grey bars is a measure of stable beam availability during 24 hours. The absence of filled bars indicates a period of no stable beams. Each green bar corresponds to an average efficiency calculated for a period of 24 hours. Reasons for lower efficiency are stop of the run during beam time to work on a sub-system and possible trigger holds due to a subsystem issuing busy for a brief period of time. The average efficiency over the whole period is $96.5 \%$.

[2] ATLAS Collaboration, ATLAS Level-1 Trigger Technical Design Report, CERN/LHCC/98-014 (1998).

[3] ATLAS Collaboration, ATLAS High-Level Trigger Data Acquisition and Controls Technical Design Report, CERN/LHCC/2003-022 (2003).

[4] R. Cranfield et al, The ATLAS ROBIN, JINST 3 (2008) T01002.

[5] The CASTOR project page at CERN. [Online]. Available: http://castor.web.cern.ch/castor

[6] Data Streaming Study Group, Report on Data Streaming in ATLAS, 09 November 2007. [Online]. Available: https://twiki.cern.ch/twiki/bin/ view/Atlas/DataStreamingReport 